\title{
Editorial
}

Journal of Innate

Immunity

Published online: November 11, 2011

DOI: $10.1159 / 000334447$

\section{Accessory Cells: No Longer Just the Bridesmaids, Now at Last the Bride}

\author{
The Nobel Prize in Physiology or Medicine 2011
}

The Nobel awards to Bruce A. Beutler, Jules A. Hoffman and Ralph M. Steinman mark the paradigm shift from lymphocytes to antigen-presenting cells (APC), often regarded as accessory to the antigen-specific arm of immunity and thus equalizing the balance between adaptive and innate immunity. Recognition of foreign antigens, via $\mathrm{T}$ and $\mathrm{B}$ cell receptors which provide diversification by somatic rearrangement, ultimately depends on germline-encoded receptors for microbial ligands, e.g. lipopolysaccharide, Toll-like receptors (TLR) and the intervention of dendritic cells (DCs) as the bridge between innate and adaptive immunity. DCs, closely related to macrophages, are specialized to activate naïve lymphocytes in association with MHC molecules, whereas macrophages are more broadly involved in homeostasis and able to activate already-primed lymphocytes. Together, these APC are widely dispersed in the body, serve as sentinels for infection and migrate to lymphoid organs where they activate or inhibit the effector functions of lymphocytes, thus regulating cellular and humoral immunity. In turn, APC are reciprocally regulated by lymphocytes, which modulate their effector functions, such as host resistance to infection, local and systemic inflammation and repair, as well as tissue injury and toxicity resulting from sepsis.

This editorial provides an opportunity to congratulate the laureates, two of whom are members of our editorial board (fig. 1, 2), and to mourn the untimely death of Ralph M. Steinman (fig. 3). It also makes it possible to place their achievements in a broader context, just over a century after the Nobel awards to Elie Metchnikoff and Paul Ehrlich for cellular and humoral immunity. The 1908 awards reconciled two opposing points of view, confirming that these are interdependent. The latest awards acknowledge the importance of the innate system as an equal partner of adaptive immunity, recognized itself by several awards during the intervening years.

It would be invidious to select a few from the many researchers who have also contributed to this revolution, but it is important to acknowledge the debt owed to earlier investigators, in this instance Charles A. Janeway, Jr., and Zanvil A. Cohn (fig. 4), who would undoubtedly have shared in this year's awards. There remain additional discoveries of major impact, in the areas of both innate and adaptive immunity, which may well feature in future awards. When the history of antigen recognition, processing and presentation comes to be written it will provide a rich subject for inquiry. Why was the discovery of TLR so long in coming when lipopolysaccharide chemistry had been studied actively over many decades? Why was the discovery of DCs, as rare but biologically potent members of the mononuclear phagocytic family, so controversial? There is an historic thread from Metchnikoff's early studies of cellular digestion, through the total deg-

\begin{tabular}{ll}
\hline KARGER & ( ) 2011 S. Karger AG, Basel \\
1662-811X/12/0041-0001\$38.00/0 \\
$\begin{array}{l}\text { Fax +41 61 306 } 1234 \\
\begin{array}{l}\text { E-Mail karger@karger.ch } \\
\text { www.karger.com }\end{array}\end{array}$ & $\begin{array}{l}\text { Accessible online at: } \\
\text { www.karger.com/jin }\end{array}$
\end{tabular}




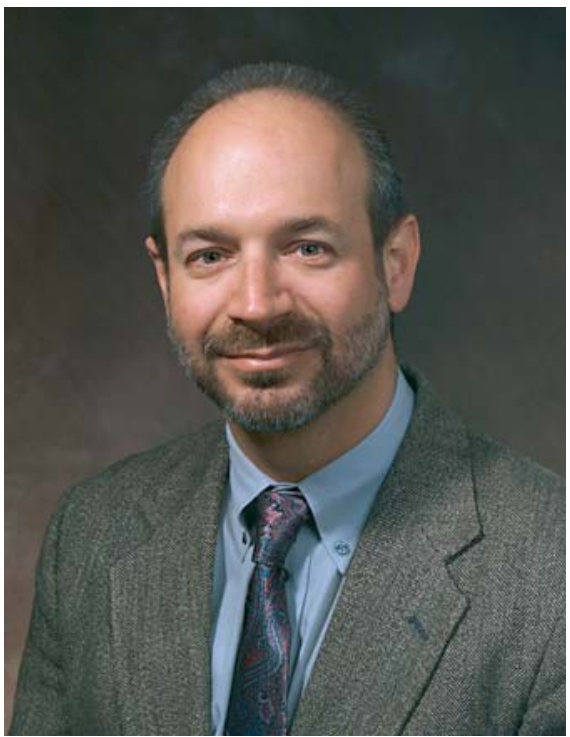

Fig. 1. Bruce A. Beutler. Courtesy of the Scripps Research Institute, La Jolla, Calif., USA.

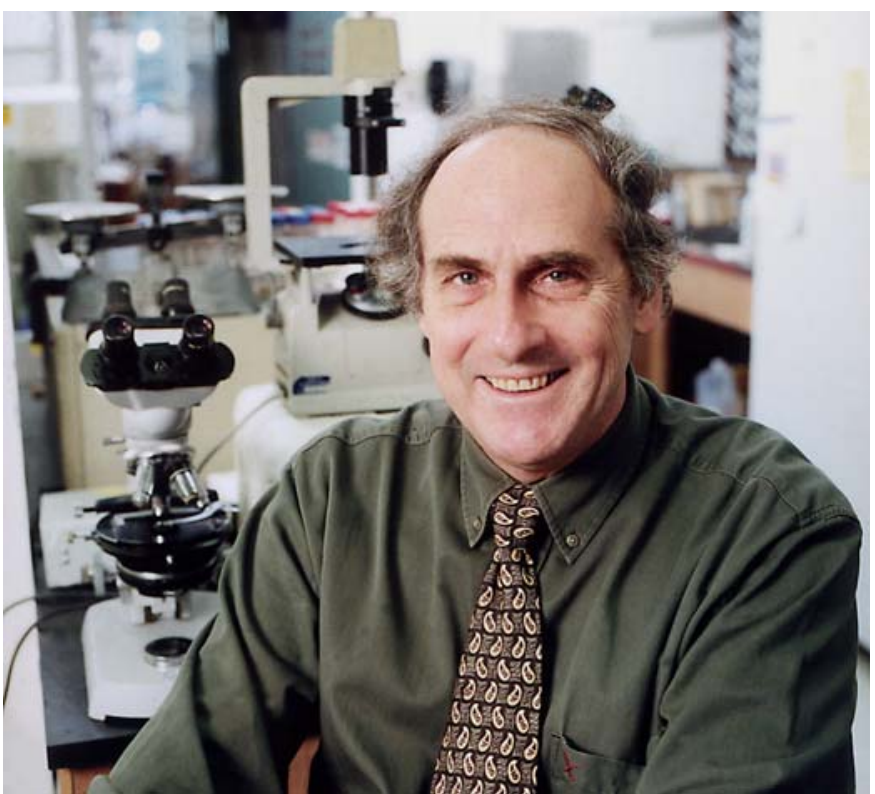

Fig. 3. Ralph M. Steinman (January 14, 1943, to September 30, 2011). Courtesy of the Rockefeller University, New York, N.Y., USA.

radation of protein antigens by macrophages to the protection of immunogenic peptides captured efficiently by DCs through MHC molecules that enables presentation at the cell surface. The current awards illustrate the value of model systems, Drosophila and mouse, which lend

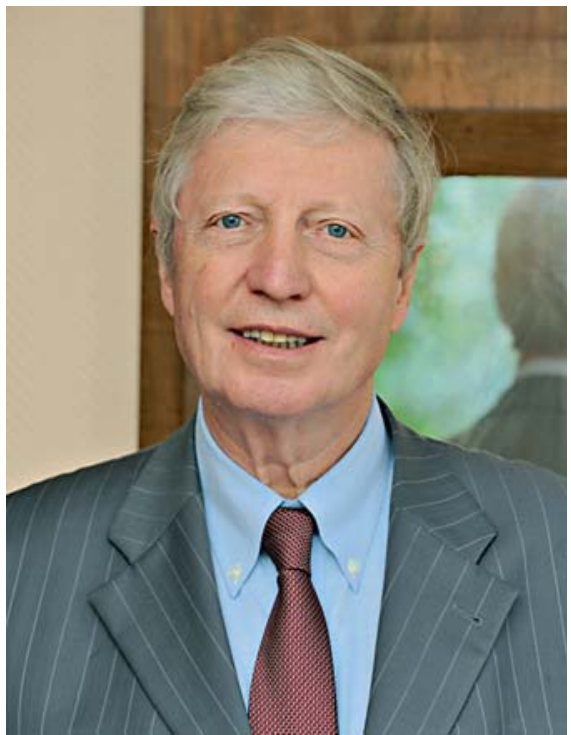

Fig. 2. Jules A. Hoffmann. Copyright: Pascal Disdier/CNRS Photothèque, Paris, France.

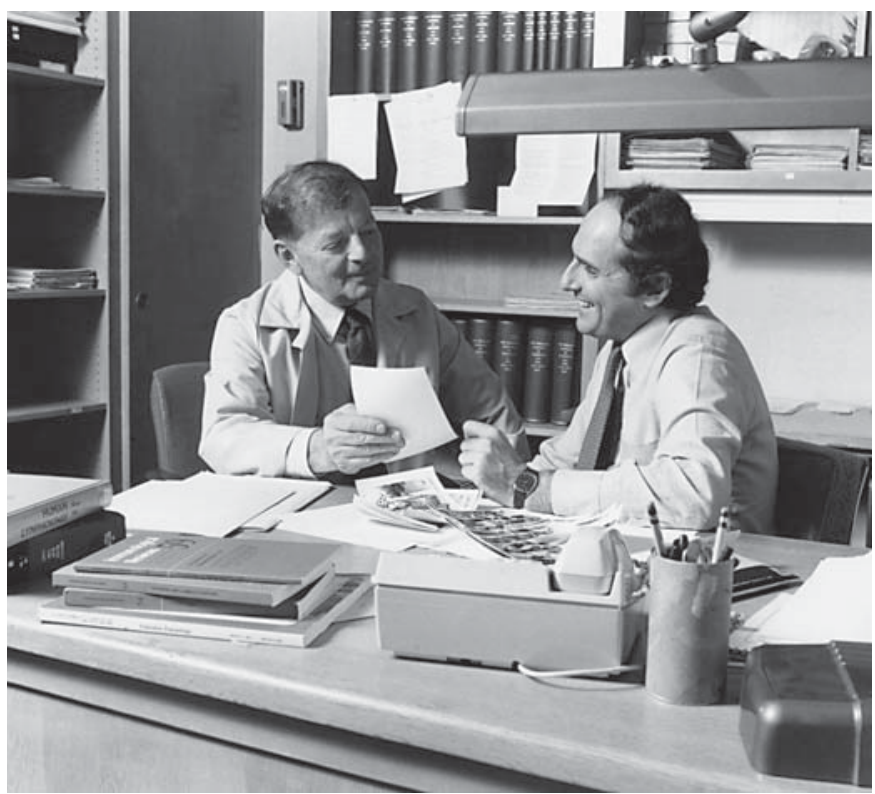

Fig. 4. Zanvil A. Cohn (November 16, 1926, to June 28, 1993) and Ralph M. Steinman (right). Courtesy of the Rockefeller University, New York, N.Y., USA.

themselves to genetic analysis, and to the exploitation of cellular immunology and microbiology in integrated studies, in vitro and in vivo.

This may not be the best time to draw attention to major issues which still need to be resolved. The complexity 
of APC differentiation and the marked heterogeneity in diverse tissue environments need to be clarified. Apart from TLR, there are additional nonopsonic receptors, such as lectins and scavenger receptors, which contribute to the homeostatic and defense functions of APC; compartmentation of other sensing molecules for microbial ligands and nucleic acids is an important aspect of intracellular infection. The rules that determine the signaling crosstalk that gives rise to activation versus suppression remain unknown. Indeed, we have not solved the central problem of immune discrimination between foreign and self, to be able to avoid the horror autotoxicus of Ehrlich, since the TLR and other sensors can react with modified or even native self molecules under certain circumstanc- es. We need to understand those instances of modified inflammation generated in response to nonmicrobial, perhaps metabolic interactions with the immune system, which contribute to cancer, atherosclerosis and Alzheimer's disease, for example.

Finally, there are some broader lessons. All the current Nobel laureates exemplify the importance of basic science, yet their research readily translates to human disease. Their discoveries are relevant to emerging infections, such as HIV/AIDS, and offer potential new therapies. The pace of discovery continues to accelerate, yet ignorance of many fundamental mechanisms and control processes remains.

Siamon Gordon, Oxford 